\title{
Apoc Social: A Mobile Interactive and Social Learning Platform for Collaborative Solving of Advanced Problems in Organic Chemistry
}

\author{
Niels Sievertsen* and Erick M. Carreira*
}

\begin{abstract}
Mobile devices such as smartphones are carried in the pockets of university students around the globe and are increasingly cheap to come by. These portable devices have evolved into powerful and interconnected handheld computers, which, among other applications, can be used as advanced learning tools and providers of targeted, curated content. Herein, we describe Apoc Social (Advanced Problems in Organic Chemistry Social), a mobile application that assists both learning and teaching college-level organic chemistry both in the classroom and on the go. With more than 750 chemistry exercises available, Apoc Social facilitates collaborative learning through discussion boards and fosters enthusiasm for complex organic chemistry.
\end{abstract}

Keywords: Advanced-semester undergraduate $\cdot$ Collaborative/Cooperative learning $\cdot$ Mobile learning $\cdot$ Organic chemistry · Virtual learning environments
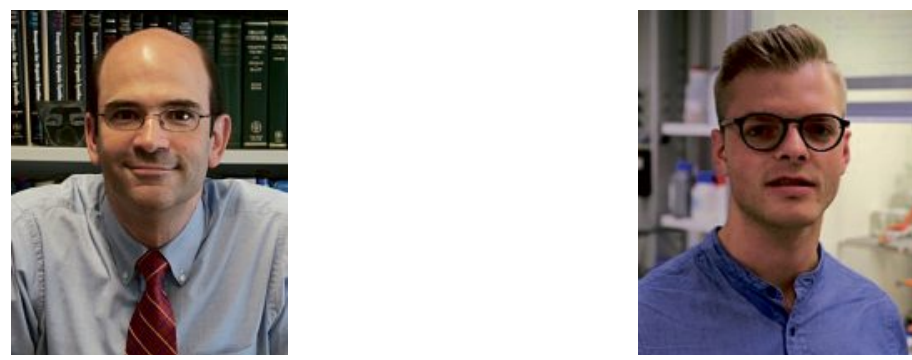

Prof. Erick M. Carreira obtained a B.S. degree in 1984 from the University of Illinois at Urbana-Champaign under the supervision of Scott E. Denmark and a PhD degree in 1990 from Harvard University under the supervision of David A. Evans. After carrying out postdoctoral work with Peter Dervan at the California Institute of Technology through late 1992, he joined the faculty at the same institution as an assistant professor of chemistry and subsequently was promoted to the rank of associate professor of chemistry in spring 1996, and full professor in spring 1997. Since September 1998, he has been professor of chemistry at the ETH Zurich in the Institute of Organic Chemistry. Since 2011, he has also been a Member of the Competence Center for Systems Physiology and Metabolic Diseases at ETH Zurich.

${ }^{\star}$ Correspondence: N. Sievertsen, Prof. Dr. E. M Carreira

E-Mail: niels.sievertsen@org.chem.ethz.ch carreira@org.chem.ethz.ch

Laboratorium für Organische Chemie, ETH Zurich

Vladimir-Prelog-Weg 3, CH-8093 Zurich

Homepage: http://www.carreira.ethz.ch
Niels Sievertsen conducted his undergraduate studies at the ETH Zurich, Switzerland. He then moved to Harvard University where he carried out his MSc thesis under the supervision of Professor A. G. Myers involving the synthesis of novel macrolide antibiotics. Having joined in spring 2016, he is currently a doctoral student in the group of Professor E. M. Carreira at the ETH Zurich. His research focus resides in the total synthesis of complex natural products.

\section{Introduction}

While methods such as textbook-based instruction and conventional lectures remain useful, relying solely on these traditional educational approaches does not fully enable active communication among students and teachers. In advanced organic chemistry courses, for example, only a small subset of the field's pioneering problems can be discussed in class due to time constraints and only few means to adequately discuss these topics in detail exist. Additionally, textbooks are both bulky and, when outdated, require expensive replacements, which may depreciate in value. A freely available mobile app organizing and augmenting existing class content with selected recent advances in organic chemistry could potentially assist teachers educate students more efficiently. As social culture changes and new technologies arise, pedagogical methods must evolve alongside them to better engage chemistry students and optimize the ways of learning for 'digital natives'.

Mobile devices - namely smartphones and tablet computers - have become deeply embedded in society as powerful, versatile, and portable tools that connect users to the world. Smartphone ownership for 18 to 24-year-olds rose from $23 \%$ to some $53 \%$ between 2009 and 2011. [1] This period marked the beginning of a new era in which owning a smartphone became ubiquitous. A recent survey in an advanced organic chemistry course for undergraduates at ETH Zürich (course identifier 5290231-00L) in September 2017 indicated that all participants owned and brought a smartphone to the first class of the fall semester. Interestingly, mobile devices are oftentimes banned in classrooms, where faculty members tend to identify them predominantly as distractions.

Given the ongoing decline in purchase price and increasing computational power of hardware, chemistry-related software could meaningfully and positively impact chemical education in high school, undergraduate and graduate studies as well as chemical professionals and teachers. Williams and Pence recently reported on 
the benefits of using smartphones and similar devices in the chemistry classroom. ${ }^{[2]}$ Mobile apps organizing and enhancing class content could potentially assist teachers reach out to their students. ${ }^{[3]}$ They would be transformed guides on the side for the additional course content provided by the software and not remain unreachable sages on the stage for many students.

Mobile apps for chemical education offer a wide range of functionalities and cover a large number of disciplines: general chemistry and inorganic, analytical, physical, biochemistry, and organic chemistry. ${ }^{[4-10]}$ Both the iOS and Android platforms provide suitable online marketplaces allowing such apps to be easily installed directly onto handheld devices. In the field of organic chemistry, apps may be grouped in five major categories: references for named reactions and study guides, molecular viewers, research-related apps, digital periodic tables, and utilities with application in the laboratory. ${ }^{[1]}$

For example, Chemistry By Design is a mobile reference for natural product syntheses catering to students in advanced semesters. ${ }^{[11]}$ The app was conceived by Jon T. Njardarson and his colleagues at Arizona University and contains a valuable set of 1746 slideshows showing total synthesis step-by-step. Furthermore, users are encouraged to choose additional published natural product syntheses and syntheses of approved pharmaceuticals and submit corresponding chemical schemes using a standardized format. A quick review process then allows such sequences to be added to Chemistry By Design and causes continuous enrichment of the app content. A range of group leaders have joined the project since its initial launch in 2012. In addition to Chemistry By Design, other similar web-based databases exist, where total syntheses can be explored. ${ }^{[12-16]}$

ReactionFlash is a collection of virtual flash cards designed to practice selected named reactions and their mechanisms developed by RELX Intellectual Properties SA. Named reactions are generally wellknown and commonly used chemical reactions within the organic chemistry community. Their mechanisms are taught to chemistry students as part of the undergraduate curriculum. ReactionFlash currently comprises about 600 named reactions. The app allows the user to order entries by difficulty and type of transformation. Individual entries can be bookmarked for later reference and a built-in quiz functionality allows to test one's knowledge. ${ }^{[17]}$ Other mobile applications almost exclusively target introductory chemistry topics and are of little use to advanced students of organic chemistry. ${ }^{[18-21]}$

Crowdsourced collaborative platforms have become a major means for sharing knowledge: Wikipedia, for example, allows instant contribution and discussion of ideas among users worldwide. ${ }^{[22,23]}$ The related forums found in StackExchange and Reddit include public discussions and simple voting systems to validate information. ${ }^{[24,25]}$ While these platforms have sections dedicated to chemistry-related topics, they are designed more as references than a practical study tool and lack a personalized curriculum for the individual user. Even photo-sharing platforms such as Instagram have been used by chemistry educators to reach students outside of the traditional brick-and-mortar classroom. ${ }^{[26]}$

The New York Times coined 2012 the 'year of MOOC'.[27,28] A MOOC (massive open online course) is an alternative to the more traditional courses offered on campuses, with all course content stored online. The non-profit start-up edX ${ }^{[29]}$ and commercial services, such as Coursera, ${ }^{[30]}$ already offer a breadth of online classes for millions of enrollees in various diverse fields of study.

It is essential to note that unlike other disciplines in science, there can be multiple correct answers to a given problem in mechanistic organic chemistry. This is what makes the field attractive and applicable to a large group of young scientists discussing and working in it. Chemical structural and reactivity space is extremely large. Thus, even when a reaction has been studied in great detail with concrete, specific mechanistic solutions available for a specific, singular example, the reaction outcome can be significantly altered by seemingly small structural changes to the reaction partners. This means that in all cases, the possibility of additional mechanisms needs to be considered.

Building on the recent advances in chemical education using handheld devices and their underlying principles, we aimed at creating collaborative exchange between students of organic chemistry to solve complex mechanistic problems. We have developed a mobile social network that would provide an efficient way for students to practice and engage organic chemistry questions on the go - whether it be on the bus to class, waiting in line at the supermarket, and so forth.

Apoc Social (Advanced Problems in Organic Chemistry Social) is a freely available, highly interactive, responsive, and community-driven learning environment. ${ }^{[31]}$ As a portable learning tool, Apoc Social is directed at generating a blended learning experience, where students themselves control time, place, path, and pace. ${ }^{[32,33]} 750$ chemistry problems now adopted in the app have been gathered from valuable contributions of Prof. D. A. Evans and members of his former research group at Harvard University, various addi- tional research groups and individuals, and the primary literature. ${ }^{[34]}$

The exercises contained within the app can be browsed by popularity, combinations of keywords, or randomly through shaking the handheld device. Every single exercise can be discussed in great detail among the users of Apoc Social. Our mobile app enables students to explore organic chemistry's current most puzzling mechanistic observations as well as landmark problems in the field. At the same time, Apoc Social is ever-expandable by assigned editors, who can easily update the problem database.

Apoc Social responds to user activity in real time and allows an algorithm-driven generation of personalized learning material for students at various levels. Tracking of one's own progress through dynamically updated statistics enables long-term motivation and allows a self-paced progression. Learners are encouraged to make use of the deeply integrated and user-driven content-moderation system to help share and approach with their solution a nurturing community both locally at ETH Zürich and around the globe.

\section{Technology Considerations}

A smartphone is commonly described as a mobile phone capable of performing many functions of a computer. Smartphones typically have a touchscreen interface, provide internet access, and carry an operating system capable of running downloaded apps. Apple iOS and Google Android are the main platforms composing $99.6 \%$ of the user base as of the fourth quarter of 2016 (iOS 17.9\%, Android $81.7 \%$ ).[35] Windows and Blackberry, in contrast, represent a negligible fraction with WindowsPhone serving about $0.3 \%$ of users and Blackberry operating system for less than $0.05 \%$ of users.

iOS and Android were chosen as target operating systems for Apoc Social, ensuring the largest user groups would be catered to. A convenient method to achieve parallel development of the mobile app on the two platforms was provided by using Ionic Framework. ${ }^{[36]}$ Ionic is a complete and open-source software development kit (SDK) for creating mobile hybrid applications. Well-known web technologies such as HTML5, CSS, and Sass can be used to create these apps instead of platformspecific APIs like those in Android and iOS, and can still be distributed through the native app stores to be downloaded on devices by leveraging Apache Cordova.[37]

By requiring user accounts, Apoc Social matches an individual's previous activity to its current cloud database, taking all ratings and comments into consider- 
ation. From these analyses, a personal curriculum can be dynamically formulated. Students are encouraged to choose their own avatar, which can be used to communicate with and follow other peers within Apoc Social (Fig. 1). Certain privacy settings can be adjusted to share all, some, or none of their activity. Students can explore the available problems by shaking their device and browse them by popularity and difficulty, or refine their search using carefully chosen categories.

Each problem page provides a detailed description and further information, such as contributing editor, a suitable reaction scheme, and often comes with suggested solutions provided by the editor. Referring to the primary literature allows interested students to read up on the given observation and to help each other solve the puzzle. The problems contained in Apoc Social further allow users to rate the problems by difficulty, decorate them with descriptive tags, bookmark or to mark them as solved. These techniques help the app follow and the instructor understand an individual's progress and determine their knowledge level.

A central component of a problem page is the problem's discussion board. Users are prompted to utilize the discussion board to think about the specific transformations together, meaning that a solution is proposed by a group rather than by an individual. This concept is facilitated by text and image submission. A simple voting system for contributions helps to rate and order students' answers and aims to reflect the level of common agreement among the user base. Connecting with peers has been simplified further by allowing users to share their individual progress with friends from within the mobile app. A global activity feed available to all users highlights interactions with problems as a live stream in real time, making it easy for any user to join a conversation or current topic in the community.

Most interactions with the app are recorded, unless the setting is explicitly switched off by the user, as anonymized data. The number of problem views, number and quality of comments, and difficulty ratings are taken into the equation when determining problem popularity and level of sophistication. These metrics are then matched with a user's progress and preferences to generate the personalized problem set.

Cloud-based storage of problems had to be implemented in order to enable dynamic access to all problems once they become available or are updated by the editors. Doing so allows small updates to the Apoc Social database to be made without having to submit an entirely new iOS or Android update, which may have average review durations between a few hours (Android) and several days (Apple iOS).

A full back-end user dashboard was developed to assist professors and other instructors to easily edit or add problems, even without prior coding experience. Additionally, the administrator can moderate users and user comments/contributions in a facile and swift manner. ${ }^{[38]}$

Instructors can use Apoc Social by creating course-specific groups to address the subsets of students enrolled in their courses in question rather than the global Apoc Social community. Within the app, educators can easily assign and highlight problems to their students.

A performance assessment can be made by the teacher in the form of statistics, which include the average percentage of problems solved and the average rating given to the current set of problems by group members. Messages can be posted to the group's details page for participants to see. The results obtained can then be used to further discuss specific problems in tu-
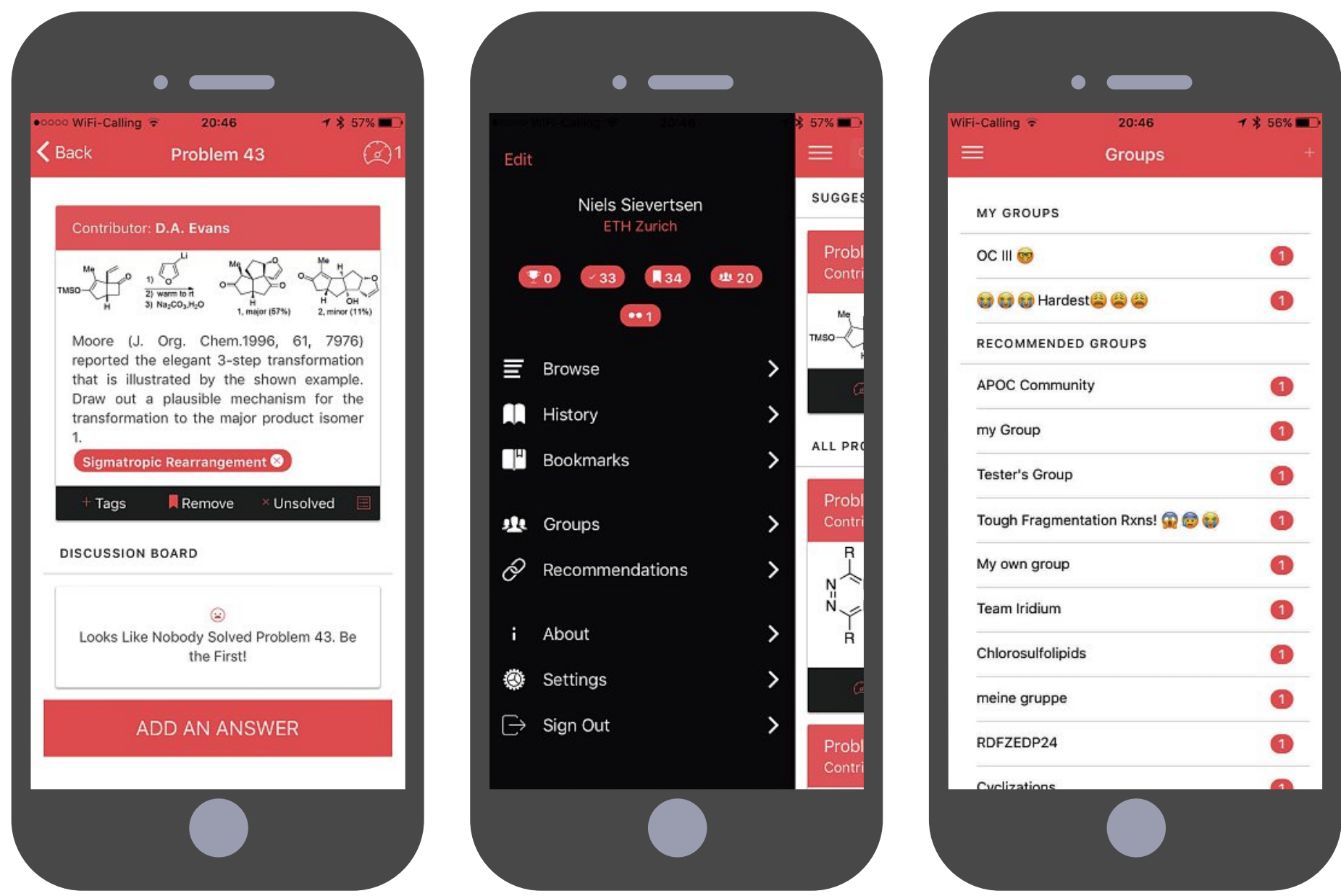

Fig. 1. Screen shot of the detailed problem view with description, reaction scheme, various options for interaction, and a discussion board (left); app navigation and personal profile (center); and user-generated group overview (right). 
torials or to dedicate more time to particular topics during lecture. Creating groups is not exclusive to teaching staff, however. Once registered with Apoc Social, users can generate groups to share interesting problems and can freely join groups of others.

\section{Implementation in the Classroom}

We sought to develop an educational supplement that would enhance and modernize the traditional teaching-learning experience in organic chemistry for advanced undergraduate students, graduate students and lecturers. Apoc Social was envisioned as an educational supplement that employs the capabilities of mobile technologies and online social connectivity and was created with an initial set of 750 advanced problems.

As a group of testers, 85 students enrolled in 'Organic Chemistry III: Introduction to Asymmetric Synthesis' (course identifier 529-0231-00L) at ETH Zürich were encouraged, but not required, to download an early version of Apoc Social to their phones. The objective of this course is to familiarize undergraduate students with fundamental principles of conformational analysis, diastereoselective carbonyl addition reactions, sigmatropic rearrangements, olefin functionalization, and applications in synthesis. Over the course of the semester, students were regularly presented with relevant questions from within the mobile app, which could then be discussed within the app or taken to problem sessions for further analysis. In return, students helped pinpoint weaknesses in prototypes and gave valuable input as to what functionality they would like to see in an organic chemistry learning app.

Over the fall 2016 and spring 2017 semesters (September 1, 2016 - August 29, 2017), Apoc Social was downloaded 2835 times across five continents. In Switzerland, an increased adoption rate was observed in the early weeks of the teaching semester and hit a maximum prior to the examination session. In the time before exams (June $1^{\text {st }}$ until August 29 $9^{\text {th }}$ ), there were 58 to 129 downloads per week with up to 48 per day. The number of sessions rose to 29 users per day and an average 57 devices were active per day. A similar spike in usage was already recorded prior to a written examination held in February 2017 where 32 active devices were observed. In preparation for examinations at the end of summer, students were again encouraged to download the app in order to assist them in their exam preparation. Notably, the app was opened 506 times in the week of August 21-27, 2017, when oral examinations for the OC III course were scheduled at ETH
Zürich. The vast majority of devices used were Apple iPhones (2454 units in the time frame of September 1, 2016 -August $29,2017)$. iPads were used to download the app 366 times. The early Apoc prototype was also installed on 15 iPods. ${ }^{[39]}$ On Android, Apoc Social was downloaded a total of 4913 times (September 1, 2016 August 29, 2017). ${ }^{40]}$

The figures representing adoption of the application put Apoc Social in a formidable position to continue to provide a fun and supportive environment, where hundreds of course-related topics are right at the students' fingertips. While using the deeply integrated and user-driven collaborative content-moderation system in the coming semesters in 'Organic Chemistry III: Introduction to Asymmetric Synthesis' (course identifier 529-0231-00L) as well as the more advanced 'Organic Synthesis: Methods and Strategies' (course-identifier 529-0233-00L) at ETH Zürich, students will help nurture a community of young chemists both locally and around the globe while improving their own confidence with advanced organic chemistry.

\section{Conclusions}

Appropriate use of the power associated with mobile teaching apps is clearly growing to become an important pillar of chemical education. As the cost for the handheld devices carrying such software and the financial burden of mobile data usage continue to drop, the percentage of students in possession of a smartphone in class will likely further increase. Understandably, those present in a chemistry classroom will routinely carry a fully stocked equivalent of a major research library in their pockets. Community-based content management, provided by mobile apps such as Apoc Social, assists in organizing knowledge and makes intriguing questions in the field available to open discussion. The social app gives an important chance to solve such organic chemistry questions as a community. Linked to the phone owner, Apoc Social can help in pointing out strengths and gaps in students' knowledge and can therefore act as a powerful learning companion for organic chemistry students at higher education institutions and a refreshing tool for professionals in the field.

\section{Acknowledgements}

We are indebted to Prof. D. A. Evans and his former research group at Harvard University for providing the majority of organic chemistry questions and, in many cases, suggested solutions. We thank the Innovedum fund at ETH Zürich (project number 1409) for generous financial support. Students enrolled in 'Organic Chemistry III: Introduction to
Asymmetric Synthesis' (course identifier 5290231-00L) during the time of development are acknowledged for testing Apoc Social at various stages. We are grateful to members of our research group at ETH Zürich for checking and categorizing the questions found in Apoc Social. Cindt LLC is acknowledged for external assistance with programming parts of the mobile application.

\section{Notes}

The authors declare no competing financial interest.

Received: September 29, 2017

[1] D. Libman, L. Huang, J. Chem. Educ. 2013, 90, 320.

[2] A. J. Williams, H. E. Pence, J. Chem. Educ. 2011, 88,683 .

[3] H. Kim, P. Chako, J. Zhao, J. K. Montclare, J. Chem. Educ. 2014, 91, 1818.

[4] S. Bandyopahdyay, B. B. Rathod, J. Chem. Educ. 2017, 94, 946.

[5] L. J. Silverberg, J. Chem. Educ. 2013, 90, 1087.

[6] J. X. H. Wong, F. S. F. Liu, H.-Z. Yu, Anal. Chem. 2014, 86, 11966.

[7] M. Montangero, J. Chem. Educ. 2015, 92, 1759.

[8] E. K. Grasse, M. H. Torcasio, A. W. Smith, $J$. Chem. Educ. 2016, 93, 146.

[9] V. D. B. Bonifaćio, J. Chem. Educ. 2012, 89, 552.

[10] L. Benedict, H. E. Pence, J. Chem. Educ. 2012, $89,492$.

[11] a) C. Dragici, J. T. Njardarson, J. Chem. Educ. 2012, 89, 1080; b) Chemistry By Design, http:// chemistrybydesign.oia.arizona.edu, accessed September 27, 2017.

[12] Prof. H. Reich's collection of natural product syntheses, https://www.chem.wisc.edu/ areas/reich/syntheses/syntheses.htm, accessed September 27, 2017.

[13] SynArchive, https://www.synarchive.com, accessed September 27, 2017

[14] Prof. D. Trauner's 'Denksport' exercises, http:// www.cup.uni-muenchen.de/oc/trauner/denksport, accessed September 27, 2017.

[15] Prof. R. Sarpong's 'Denkport' exercises, http:// www.cchem.berkeley.edu/rsgrp/schedule-current.html, accessed September 27, 2017.

[16] Prof. T. Fukuyama's Group Meeting Problems, http://www.f.u-tokyo.ac.jp/ fukuyama/index-e. htm, accessed September 27, 2017.

[17] a) ReactionFlash for iOS, https://itunes.apple. com/us/app/reactionflash/id432080813, accessed September 27, 2017; b) ReactionFlash for Android, https://play.google.com/store/apps/ details?id=ch.reaxys.reactionflash \&hl=en, accessed September 27, 2017.

[18] J. Winter, M. Wentzel, S. Ahluwalia, Chem. Educ. 2016, 93, 1657.

[19] Mahjong Chem, http://www2.stetson.edu/mahjongchem/, accessed September 27, 2017.

[20] Sokobond, http://www.sokobond.com, accessed September 27, 2017.

[21] ChemCrafter, http://assets.chemheritage.org/ ChemCrafter/, accessed September 27, 2017.

[22] H. E. Pence, A. J. Williams, R. E. Belford, 'Chemistry Education: Best Practices, Opportunities and Trends', Eds. J. GarcíaMartínez, E. Serrano-Torregrosa, Wiley, Weinheim, 2015, p. 702.

[23] Wikipedia Chemistry Portal, https://en.wikipedia.org/wiki/Portal:Chemistry, accessed September 27, 2017.

[24] Chemistry Reddit, https://www.reddit.com/r/ chemistry/, accessed September 27, 2017. 
[25] Chemistry StackExchange, https://chemistry. stackexchange.com, accessed September 27, 2017.

[26] A. L. Korich, J. Chem. Educ. 2016, 93, 1134.

[27] N. J. Pienta, J. Chem. Educ. 2013, 90, 271.

[28] L. Pappano, The New York Times, Nov 2, 2012, http://www.nytimes.com/2012/11/04/education/ edlife/massive-open-online-courses-are-multiplying-at-a-rapid-pace.html, accessed September 27, 2017.

[29] edX course offerings, https://www.edx.org, accessed September 27, 2017

[30] Coursera offerings, https://www.coursera.org, accessed September 27, 2017.

[31] a) Apoc for iOS, https://itunes.apple.com/ch/ app/apoc-advanced-problems-in-organicchemistry/id1019335489, accessed September 27, 2017; b) Apoc for Android, https:// https:// play.google.com/store/apps/details?id=com. ionicframework.eq4303509\&hl=en, accessed Septemner 27, 2017; c) Apoc Social for iOS, https://itunes.apple.com/ch/app/apoc-socialproblems-in-organic-chemistry/id1180554411, accessed September 27, 2017; d) Apoc Social for Android, https://play.google.com/ store/apps/details? id=com.nielssievertsen. apocsocial\&hl=en, accessed September 27, 2017; e) Apoc Social project details at Innovedum, https://ww2.lehrbetrieb.ethz.ch/ ivy/pro/Innovedum/Innovedum/showProject. jsp?id=1409\&lang=en, accessed September 28, 2017; f) Code for Apoc and Apoc Social is accessible for free at GitHub, https://github. com/nielssievertsen, accessed September 28, 2017.

[32] N. J. Pienta, J. Chem. Educ. 2016, 93, 1.

[33] M. K. Seery, C. O'Connor, 'Chemistry Education: Best Practices, Opportunities and
Trends', Eds. J. García-Martínez, E. SerranoTorregrosa, Wiley, Weinheim, 2015, p. 652

[34] a) Prof. D. A. Evans group website, http://evans. rc.fas.harvard.edu, accessed September 27, 2017

[35] J. Vincent, The Verge, Feb 16, 2017, https:// www.theverge.com/2017/2/16/14634656/android-ios-market-share-blackberry-2016m, accessed September 27, 2017.

[36] Ionic Framework, https://ionicframework.com, accessed September 27, 2017.

[37] Apache Cordova, https://cordova.apache.org, accessed September 27, 2017.

[38] Apoc Social administrator's dashboard, http:// api.apoc.social, accessed September 27, 2017.

[39] iTunes Connect, https://itunesconnect.apple. com, accessed September 27, 2017.

[40] Google Play Store Developer Console, https:// play.google.com/apps/publish, accessed September 27, 2017. 